\title{
THE INVESTIGATION OF CORRELATIONS OF PAIN SCORING SYSTEMS USED IN EVALUATING ANALGESIC EFFECTS AFTER ABDOMEN OPERATIONS
}

\author{
O Kiran, T Mıngır, N Turgut \\ Ministry of Health Okmeydani Training and Research Hospital, Department of \\ Anesthesiology and Intensive Care, Istanbul, Turkey
}

Objective: It was planned to investigate the correlation of Numerical pain scale (NRS) and Prince Henry pain scale (PHPS) scoring systems used to assess postoperative pain levels.

Materials and Methods: Seventy-five patients who underwent unilateral inguinal hernia repair from ASA I-III over the age of 18 years were included in the study. Transversus Abdominis Plan (TAP) Block was applied to each patient at the end of the operation for standard postoperative analgesia following standard general anesthesia. Demographic data of the patients and postoperative 0., 2., 4., 6., 8., 12.hour nausea at time, presence of vomiting, need for additional analgesia, NRS and PHPS values were compared.

Results: Demographic characteristics of the cases (Table 1.) and postoperative analgesic usage times (Table 2.) are also seen. Nausea was observed in $20 \%(n=15)$ and vomiting in $2.7 \%(n=2)$. There were statistically significant difference between NRS values and PHRS at 0., 2., 4., 6., 8. and 12. hours (respectively $p$ $=0,001, p<0,01$, Figure 1., $p=0,001, p<0,01$, Figure 2.). The relationship between the two pain scores was assessed by Spearman's correlation analysis and ICC, and the relationship between the two pain scores was found statistically significant in both cases. PHPS scores of the cases were found at time $0(r=$ $0,773 ; p<0,01)$ among the NRS scores; $2^{\text {nd }}$ hour $(r=0,711 ; p$ $<0,01)$; At $4^{\text {th }}$ hours $(r=0.725 ; p<0,01)$; At $6^{\text {th }}$ hours $(r=0,640 ; p$ $<0,01)$; Positive correlations were found statistically significant at $8^{\text {th }}$ hour $(r=0,786 ; p<0,01)$ and $12^{\text {th }}$ hour $(r=0,743 ; p<0,01)$ (Table 3.).

Conclusion: PHPS also provides an effective assessment, such as NRS, in assessing pain after abdominal operations. We believe that PHPS can provide a more objective assessment especially during the early postoperative period. However, larger, heterogeneous sampling studies are needed.

Table 1: Distribution of descriptive properties

\begin{tabular}{|l|l|l|l|}
\hline & & Min-Max (Median) & Average \pm Ss \\
\hline Age $($ Years $)$ & & $22-80(55)$ & $54,07 \pm 15,13$ \\
\hline BMI $\left(\mathrm{kg} / \mathrm{m}^{2}\right)$ & & $19,1-29,9(26,6)$ & $26,45 \pm 2,05$ \\
\hline ASA & & $1-3(1)$ & $1,57 \pm 0,70$ \\
\hline & & $\mathrm{n}$ & $\%$ \\
\hline \multirow{3}{*}{ Gender } & Female & 5 & 6,7 \\
\cline { 2 - 4 } & Male & 70 & 93,3 \\
\hline \multirow{3}{*}{ ASA } & I & 41 & 54,7 \\
\cline { 2 - 4 } & II & 25 & 33,3 \\
\cline { 2 - 4 } & III & 9 & 12,0 \\
\hline
\end{tabular}

Table 2: Usage of analgesics according to postoperative time

\begin{tabular}{|r|c|c|}
\hline analgesic usage & $\mathrm{n}$ & $\%$ \\
\hline Start & 24 & 32,0 \\
\hline $2^{\text {nd }} \mathrm{h}$ & 11 & 14,7 \\
\hline $4^{\text {th }} \mathrm{h}$ & 8 & 10,7 \\
\hline $6^{\text {th }} \mathrm{h}$ & 4 & 5,3 \\
\hline $8^{\text {th }} \mathrm{h}$ & 3 & 4,0 \\
\hline $12^{\text {th }} \mathrm{h}$ & 0 & 0,0 \\
\hline
\end{tabular}

Table 3: Relationships and ICC Compliance between Numerical Rating Score and Prince Henry Pain Score

\begin{tabular}{|l|l|l|l|l|l|}
\hline & \multicolumn{5}{|c|}{ Numerical Rating Score -Prince Henry Pain Score } \\
\cline { 2 - 7 } & $\mathrm{r}$ & $\mathrm{p}$ & $\mathrm{ICC}$ & $\% 95 \mathrm{Cl}$ & $\mathrm{p}$ \\
\hline Start & 0,773 & $0,001^{* *}$ & 0,696 & $0,558-0,796$ & $0,001^{* *}$ \\
\hline $2^{\text {nd }} \mathrm{h}$ & 0,711 & $0,001^{* *}$ & 0,698 & $0,561-0,798$ & $0,001^{* *}$ \\
\hline $4^{\text {th }} \mathrm{h}$ & 0,725 & $0,001^{* *}$ & 0,680 & $0,537-0,785$ & $0,001^{* *}$ \\
\hline $6^{\text {th }} \mathrm{h}$ & 0,640 & $0,001^{* *}$ & 0,615 & $0,452-0,738$ & $0,001^{* *}$ \\
\hline $8^{\text {th }} \mathrm{h}$ & 0,786 & $0,001^{* *}$ & 0,774 & $0,664-0,851$ & $0,001^{* *}$ \\
\hline $12^{\text {th }} \mathrm{h}$ & 0,743 & $0,001^{* *}$ & 0,630 & $0,471-0,749$ & $0,001^{* *}$ \\
\hline
\end{tabular}

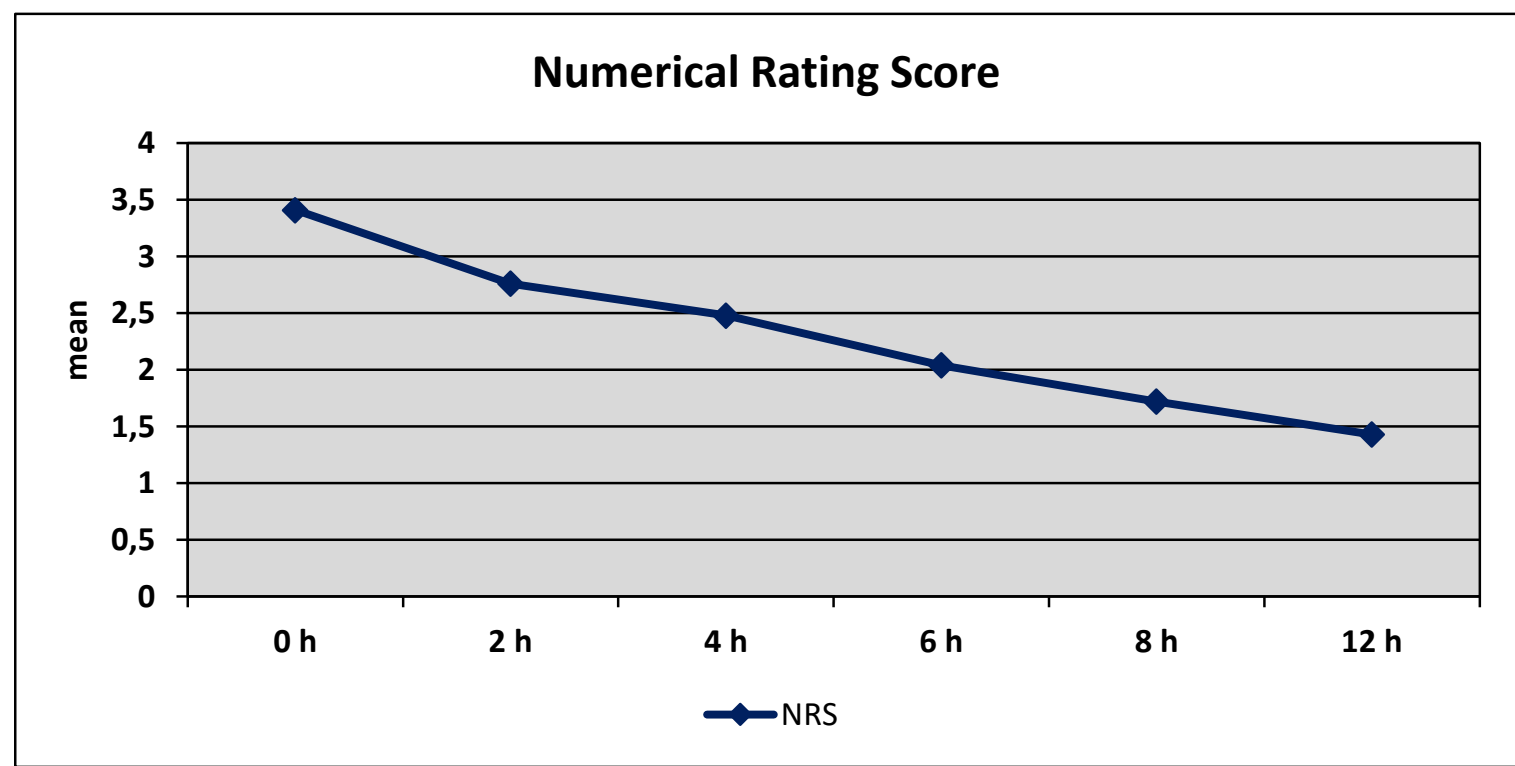

Figure 1.Numerical Rating Score distribution by time

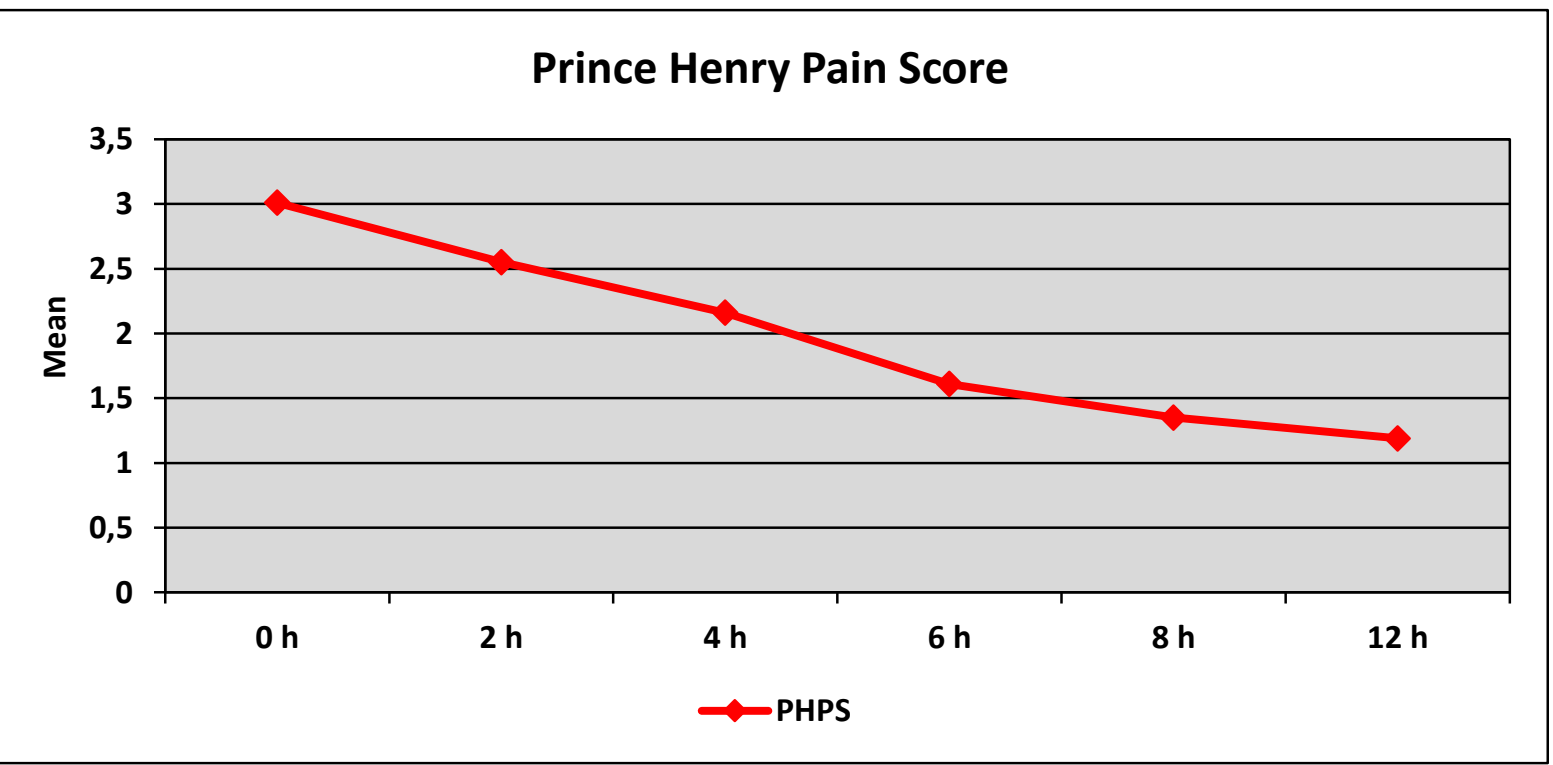

Figure 2. Prince Henry Pain Score distribution by time 